\title{
ADVANCED TARGET CONCEPTS FOR PRODUCTION OF RADIOACTIVE IONS AND NEUTRINO BEAMS *
}

\author{
H. L. Ravn, ${ }^{* *}$ \\ The ISOLDE collaboration and the neutrino-factory working group. \\ EP-Division, CERN, CH-1211 Geneve 23 Switzerland \\ Proceedings of the $14^{\text {th }}$ International Conference on Elec tromagnetic \\ Isotope Separators and Techniques Related to their Applications, \\ Victoria, Canada, May 2002. \\ Nuclear Instruments and Methods B.
}

PACS: 29.25.-t, 29.25.Rm, 29.25.Dz

Keywords: Liquid metal, Liquid Mercury, High power targets, Radioactive ions, Pion, Muon, Neutrinos

\begin{abstract}
The 1-20 MW of proton beam power which modern accelerator technology put at our disposal for production of intense secondary beams presents a major technically challenge to the production targets. A conceptual design is presented for a high-power pion production target and collection system, which was originally suggested to be
\end{abstract}

\footnotetext{
${ }^{*}$ Support ed by the EU RTD project EURISOL (HPRI-CT -2001-500001)
} 
used as the source for the proposed CERN muon-neutrino factory. It will be shown that the major parts of this target could also serve as an efficient spallation neutron source for production of ${ }^{6} \mathrm{He}$ and fission products in the two-step converter-target concept.

The heart of the system consists of a free surface Mercury jet with a high axial velocity, which allows the heat to be carried away efficiently from the production region. For the neutrino factory the secondary pions are collected and injected into the pion decay-channel by means of a magnetic horn. For the radioactive ion-beam facility the $\mathrm{Hg}$-jet is surrounded by the high-temperature ISOL production-target.

The suggested mechanical layout and technical parameters of the Hgjet, ISOL target, horn and cooling system are discussed. The critical issues are identified and a description of the $\mathrm{R} \& \mathrm{D}$ program designed to provide experimental proof of the principle as well as providing engineering parameters is given.

\section{INTRODUCTION}

The most powerful proton beams are very attractive as drivers for various facilities for production of beams of secondary particles like spallation neutron sources, $[1,2]$, neutrino factories [3] and the planned radioactive nuclear beam accelerator EURISOL $[4]$.

The destructive effects on the target equipment and its surroundings caused by the megawatt-scale power and radiation dissipated by such intense beams represent a major challenge that identifies the target station and its support laboratories as one of the most crucial and possibly cost driving items of such facilities. The successful accelerator developments, which allow the construction of machines capable of producing intense particle beams in the $\mathrm{GeV}$ range with a total average power of more

\footnotetext{
** E-mail: Helge.Ravn@cern.ch
} 
than 5MW have not yet been followed by similar investments in the development of the advanced targets needed in order make use of this power for producing secondary beams with intensities that may be several orders of magnitude greater than those available at facilities presently in operation.

While the spallation neutron sources may ease their heat transfer problem by depositing the beam power in large amounts of target material the neutrino factories and the radioactive nuclear beam facilities need target volumes of orders of magnitude smaller in order to efficiently release the particles to be captured and accelerated. In the neutrino factories it is planned to make intense beams of muon neutrinos obtained by decay of accelerated and stored muons produced from the decay of pions. In the RIB facilities emphasis is put on the production of intense beams of the most short-lived fission products for which neutrons would be the best driver particles. Both facilities have in common the fact that they need a compact converter target either surrounded by a pion collector or an ISOL target. Recently a very interesting combination of ISOL and a neutrino factory has been suggested [5]. Intense beams of both flavours of electron neutrinos (beta beams) may be obtained from short-lived beta emitting nuclei accelerated and stored in the existing CERN accelerator complex [6].

Primary converter targets made out of solids may still function up to relatively high power densities but seem to have lifetime problems at the ultimate power densities. Liquid metal target and heat transfer technology have been chosen by the other above mentioned projects as the most promising direction which may lead to use of even higher driver beam intensities [7]. This paper describes and discusses some recent ideas of advanced high power targets for production of intense beams of radioactive ions in neutrino factories and radioactive nuclear beam accelerators. 


\section{TARGETS FOR RADIOA CTIVE NUCLEAR BEAMS}

A little more than 50 years ago the first Radioactive Ion-Beam (RIB) experiment was successfully demonstrated at the Niels Bohr Institute [8]. Well ahead of his time he invented the Isotope Separator OnLine (ISOL) concept to address fundamental lepton interactions namely the beta neutrino angular correlation. The experiment contained a number of innovations like the two stage target-technique in which the deuteron driver beam produced neutrons in a primary converter-target to be used in a secondary target to produce gaseous fission products that in turn were ionised and accelerated. Since then the ISOL technique has been successfully used in a number of low energy and intensity nuclear physics facilities. During this time a large amount of target techniques have been developed and successfully used to produce low energy beams of more than 600 unstable isotopes from $70 \%$ of the elements. While on-line to reactors and accelerators providing a variety of driver particles it was demonstrated that a large potential for further improvements with regard to higher intensities, higher energies and more radioactive particle beams existed. In fact the time now seems ripe to readdress neutrino physics by means of the ISOL technique as discussed in section 3.5.

\subsection{Targets irradiated directly by the proton beam}

Proton beams $>=1 \mathrm{GeV}$ with intensities up to $4 \mu \mathrm{A}$ have, for 30 years, routinely been in use at CERN/ISOLDE. Since these targets need to be electrically heated in addition to the power deposited by the charged beam it is safe to assume that these techniques can all be used for proton beams intensities of 10-20 $\mu \mathrm{A}$. The use of higher proton intensities directly onto the targets requires their active cooling. The efficiency of cooling of the targets presently in use depends strongly on target material properties like density, heat conductivity and volume. These methods will have to be developed 
individually for each element. Only one such target has been thoroughly engineered and optimised for its high power properties. By means of radiation cooling the RIST/ISOLDE Tantalum foil target for Li production [9] could be shown to dissipate up to $30 \mathrm{~kW}$ of power i.e. the power deposited by a $1 \mathrm{GeV}$ proton beam of $100 \mu \mathrm{A}$ intensity.

It is concluded that present technology limits the proton intensity to a maximum of $100 \mu \mathrm{A}$ and this should be the design aim of at least one target station at EURISOL.

\subsubsection{Two stage targets for neutron reactions}

The EURISOL study of the possibilities of using the production potential of an up to $4 \mathrm{MW}$ of $1 \mathrm{GeV}$ proton beam shows clearly that new technologies have to be introduced. The concept of choice is to go back to the charged particle to neutron converter technique originally used by Kofœd Hansen et al. As advocated by J. Nolen [10] the power of the charged driver beam can be dissipated and disposed of in a primary target and the produced neutrons used to generate beams of fission products in an ISOL target and ion-source unit with known technology without overheating it. Such very compact and high power converter targets that allow efficient capture of the produced pions have already been discussed in a series of workshops on Neutrino factories $[11,12,13]$ held over the last few years. As a matter of fact these high $\mathrm{Z}$ targets described in section 3 are also prolific spallation neutron sources. It is therefore suggested that they should also be used in the EURISOL high power target station by replacing the surrounding pion collector with the fissioning target. Since

EURISOL plans to use a CW driver beam the destructive thermal expansion waves that seriously complicates the design of the pion targets are absent. 


\section{TARGETS FOR NEUTRINO FACTORIES}

After the target the pions are collected and injected into the decay-channel by means of a magnetic horn or superconducting solenoid that need to surround the target and preferably to also accommodate the spent beam absorber. The pions emerging from the target have a very large divergence and energy spread and decay rapidly into 2.2 $\mu$ s muons. In order to capture, accelerate and store them they have to be phase rotated and cooled.

In order to achieve this the pion precursors have to be produced by trains of very short proton bunches (nanoseconds). The driver beam chosen in the CERN scenario is obtained by accumulating and bunching the beam from the proposed Super Conducting Linac (SPL) [14]. It will deliver a proton beam of $2.2 \mathrm{GeV}$ that is pulsed at $50 \mathrm{~Hz}$ with $\sim 10^{14}$ protons per pulse and $4 \mathrm{MW}$ average power. Each pulse train lasts only $3.3 \mu$ s and consists of 140 bunches.

The key problem for the target is the initial pressure waves induced in any condensed matter by the $\mu$ s short proton pulse, which deposits about $100 \mathrm{~kJ}$ heating power. They eventually exceed the yield strength of any solid target or tubes containing liquid and cause them to break down mechanically after a short time.

Although the free surface $\mathrm{Hg}$-jet target located inside the magnetic horn for pion collection has been chosen as the CERN base line muon production system a few interesting solid target alternatives also under study are briefly discussed below. It is also shown that a number of target and muon cooling problems may be solved if the neutrino factory is based on electron neutrinos derived from an ISOL beam using known target technology [15]. 


\subsection{Solid moving targets}

New innovative techniques seem to be needed in order to dispose of the power generated in the small volume of a pion production target where local densities of up

to $100 \mathrm{~kW} \mathrm{~cm}^{-3}$ may be encountered. The conce pt in which solid metal target material is re-circulated rapidly in the beam in order to spread the power and damage into a large amount of material is that most frequently used in the facilities operating at present.

A number of ideas of extending this concept to higher intensities were presented at the NuFact99 and 01workshops [11,12]. A particularly elegant solution of a magnetically levitated and driven high $\mathrm{Z}$ target ring cooled by radiation is now under study in order to verify the simulations and determine engineering parameters [16]. Recent tests in the low power ISOLDE beam of radiation cooled solid Ta converter targets have shown severe deformation as discussed in Ref. [17].

\subsection{Stationary target in granular form}

Recently Sievers [18] has shown that dividing the solid into small granules may grossly reduce the damage caused by the thermally induced shocks observed in solid targets. Since each granule only absorbs a small fraction of the beam power and are not mechanically coupled to the others the dmaging peak stresses observed in the oscillation of a single body are reduced.

Owing to the high surface to volume ratio of the ensemble of the granules the high power densities may be handled by the conventional technique of direct Helium or water-cooling. The crucial problems are heat transfer, material stress, fatigue, radioactivity, radiation damage, in particular of any windows of the target container and cooling media confinement. 


\subsection{Free surface Hg-jet target}

The solutions mentioned above may provide only limited performance but can be useful as a first approach at lower powers.

As target for the maximum beam powers a jet of Mercury is proposed. The essential feature is that the target is presented to the proton beam as a continuous free surface liquid-metal-jet with $\sim 20 \mathrm{~mm}$ diameter and an axial velocity of $<30 \mathrm{~m} / \mathrm{s}$. It is rapidly reformed after each beam pulse that only scatters the liquid due to the above mentioned thermal expansion wave and allows the heat to be carried away efficiently from the production region without need for nearby beam windows.

Like in the spallation neutron sources, the metal Mercury has been chosen as the most promising high $\mathrm{Z}$ target and coolant material but it could be replaced by several other metals or alloys including low $\mathrm{Z}$ elements.

The following additional advantages of a $\mathrm{Hg}$-jet target should be noted:

- High pion yield (high Z)

- High source brightness (high density)

- Flowing liquids have excellent power handling capabilities

- No water radiolysis

- Liquid at ambient temperature (no liquid-to-solid phase change issues)

- Minimal waste stream (compared to solid alternatives) since the $\mathrm{Hg}$ is continuously reused.

The majority of the radioactive reaction products may even be concentrated and removed from the $\mathrm{Hg}$ by distillation No confinement tubing (free surface jet)

No need for near by beam windows (differential pumping confinement) 
The cavitation and disruption of a molten metal by the proton beam interaction was already noted in ISOLDE $\mathrm{Pb}$ targets [19]. A series of experiments performed with the Brookhaven $24 \mathrm{GeV}$ beam [20] and the ISOLDE $1.4 \mathrm{GeV}$ beam [21] proton beams allowed the confirmation and detailed study of the effect of stress waves in liquid metals while simulating power densities at $1 / 100$ of the ultimate power density and $1 / 10$ of the needed jet speed in the neutrino factory.

By means of high-speed digital cameras the break up of the $\mathrm{Hg}$-jet into droplets by the proton beam induced pressure wave propagating in both static $\mathrm{Hg}$ baths and free flowing $\mathrm{Hg}$-jet were studied. It was performed with only a few proton bunches and gave valuable data on in-beam $\mathrm{Hg}$ handling.

The data suggests the following tentative conclusions: There is no visible propagation of the expansion wave in the axial jet direction outside the short length of maximum power deposition. The droplet speed was lower in the jet $(\sim 20 \mathrm{~m} / \mathrm{s})$ than in the static test $(\sim 40 \mathrm{~m} / \mathrm{s})$ but seemed, over the short intensity range studied, to be proportional to the number of protons in the bunch. In conclusion the BNL-CERN in beam static and jet test revealed no showstopper for the Hg-target concept.

As seen from the schematic layout of such a target in Figure 1 [22] the protons are injected through an annular nozzle co-linearly into a free surface jet. Reynolds number of $10^{6}$ for a $20 \mathrm{~mm} \mathrm{30m/s} \mathrm{Hg} \mathrm{jets} \mathrm{indicate} \mathrm{that} \mathrm{the} \mathrm{flow} \mathrm{is} \mathrm{highly} \mathrm{turbulent}$ and the $\mathrm{Hg}$ jet will rapidly break up into a spray of droplets. This should have the same advantage of damping the expansion wave as described by Sievers [18] for granular solid targets discussed in section 3.2. The high droplet speeds observed by Fabich et al. [21] may thus be reduced. Also shown in Figure 1 is the magnetic horn pion collector that in the case of EURISOL use would be replaced by the ISOL target material as shown by Nolen [10]. 


\subsection{Pion collector}

In order to maximize the secondary pion collection efficiency the target needs to be surrounded by a large acceptance pion collection and focusing system.

Rather than using a super conducting $20 \mathrm{~T}$ solenoid as suggested in the US projects [23] which has the advantage of providing adequate space for the metal jet, the spent beam absorber and their plumbing, it is envisaged at CERN to use a pion collection system based on the azimuthally magnetic fields generated between the coaxial conductors of a magnetic horn. This technology traditionally used at CERN for focusing secondary particles [24] has the advantage that the parts exposed to the beam are rather simple, inexpensive and can be radiation hard. The horn should be designed to focus particles emitted at large angle, and with a momentum range of 200-400 $\mathrm{MeV} / \mathrm{c}$, from a target of a diameter of $20 \mathrm{~mm}$. In the case of the Neutrino Factory, the beam coming from the horn enters directly into a $60 \mathrm{~cm}$ diameter solenoid of the decay channel.

The hor $\mathrm{n}$ [25] is compact in the longitudinal dimension $(1 \mathrm{~m})$, while the large radial dimension is quite remarkable compared to traditional designs. This choice was dictated by the particular pion spectrum produced by the $2.2 \mathrm{GeV}$ proton beam. Most of the useful particles exit the target radially, with a typical transverse momentum of $250 \mathrm{MeV} / \mathrm{c}$. The performance of this horn is currently under study, but preliminary results show, the collection efficiency to be comparable to a $20 \mathrm{~T}$ solenoid. Limiting factors for this technology are found in the waist region of the inner conductor. It needs to have a diameter of minimum 5 to $8 \mathrm{~cm}$ in order to accommodate the target and its plumbing. The Joule losses in a waist of this diameter raise concerns about cooling followed by the lifetime limitation caused by the magnetic forces of the high current pulse and the possible weakening of the material caused by radiation damage 
A third possibility of pion collection, which has received some attention [26], is to send a high electrical current pulse directly through the molten metaljet target so that it also acts like a Lithium lens. Since the Joule losses were found to be comparable to the beam heating this method might require unrealistically high metal jet speeds.

\subsection{ISOL target for beta beams}

The alternative use of accelerated and stored short-lived radioactive ions as precursors for intense, pure electron-neutrino beams can be based on well-known ISOL techniques [15] used today in various RIB facilities. For the nuclei relevant $\mathbf{b}$ neutrino sources which are formed in high cross-section reactions with half-lives in the range of $100 \mathrm{~ms}$ to $10 \mathrm{~s}$ the prospects for achieving the needed intensities are particularly promising.

This method for production of electron neutrinos has the following technical advantages over the method for production of muon neutrinos:

1. Neutrinos of only one flavour are produced at a time

2. For a majority of the chemical elements the RIB production is a known technique.

3. No charged particle collection and cooling system is needed since the products are extracted from ion sources with good emittance ( 2 to $50 \pi \mathrm{mm} \mathrm{mrad}$ ).

4. The overall efficiency of transforming the nuclei of interest, produced in the target, into a beam is $>10 \%$.

5. The MW of spent protons can be absorbed at a location chosen out of the beam trajectory and far from the target and any accelerator component.

6. It might even be imagined to use part of the by-products consisting of pions and $150 \mathrm{GeV} / \mathrm{A}$ daughter nuclei 
As an example the principles and electron neutrino intensities which may be obtained from such an ISOL target station optimised for production of ${ }^{6} \mathrm{He}$ as proposed by Zuchelli [5] is discussed below.

The radioactive beam intensity that may be obtained from such a target station is determined by Eq. $2[27,28]$.

$$
\mathrm{I}=\sigma \Phi \quad \mathrm{N} \varepsilon_{1} \varepsilon_{2} \varepsilon_{3},
$$

Where $\sigma$ is the formation cross-section for the nuclear reactions of interest, $\Phi$ the primary-beam intensity, $\mathrm{N}$ the usable target thickness, $\varepsilon_{1}$ the product release and transfer efficiency, $\varepsilon_{2}$ the ion-source efficiency and $\varepsilon_{3}$ the delay transfer efficiency due to radioactive decay losses. The production of this rare gas isotope is particularly favourable and can be produced by a variety of methods at ISOLDE. Since $\varepsilon_{3}$ is known to be high very large $\mathrm{BeO}$ targets may be built that could efficiently surround a spallation neutron source [29] so that the high production cross section of the $n, \alpha$ reaction can be used.

In Table 1 the parameters permitted by present day technology are given for the isotope ${ }^{6} \mathrm{He}$ precursor of $\forall_{e}$ that present technology allow to use today somewhat lower yield of the $v_{e}$ precursor ${ }^{18} \mathrm{Ne}$ may also be obtained from spallation of a $\mathrm{MgO}$ target. In addition the table shows the ${ }^{6} \mathrm{He}$ expected to be reached after successful development work planned by EURISOL.

\section{CONCLUSION AND OUTLOOK}

Laboratories for molten metal technology and horn development have been set up at CERN in which many of the skills needed for designing a 4MW target station are represented. Collaboration between European laboratories has started and a Eur opean Muon Concertation and Overview Committee (EMCOC) has been formed. The first experiments with Hgjets has not uncovered any showstopper and manufacture of a prototype horn has started. A satisfactory solution for the location and type of the 
beam dump without causing excessive losses of muons remains to be found. The crucial engineering parameters determined by this program should allow a choice to be made from the various proposed technologies. The effort needed to develop such high power targets calls for further cross field collaboration.

The $R \& D$ program could be as follows:

- Study the hydrodynamics of a full scale jet (jet stability, velocity and pumping system)

- Continue the in beam Hg-trough studies at $1.4 \mathrm{GeV}$ PS-Booster beam at 1/5 of the NuFACT power density

- Start engineering study of the integration of the target plumbing in the horn or in a concentric ISOL target

- Design and build a $400 \mathrm{kA}$ pulse current generators to drive the magnetic horn at $50 \mathrm{~Hz}$

- Continue the studies of alternative target concepts like radiation cooled solids and the water cooled granular target

- Modeling of the proton beam induced expansion wave on a liquid Hg column and a liquid $\mathrm{Hg}$ spray

- Calculation and measurement of heat transfer coefficient of the gas or watercooled granular target consisting of solid spheres.

\section{REFERENCES}

[1] G. S. Bauer et al., The European Spallation Source Study, vol III, The ESS Council, ESS-96-53-M, 1996.

[2] L. K. Mansur et al., J. Nucl. Materials, 296 (2001) 1.

[3] P. Gruber et al.,The study of a European Neutrino Factory Complex CERN report to be published. 
[4] J. Vervier, Status of the EURISOL Programme, this conference.

[5] P. Zucchelli, A novel concept for a anti electron Neutrino Factory, to be published in Phys. Lett. B.

[6] M. Lindroos, Future plans at ISOLDE, this conference.

[7] R. B. Palmer et al., Nucl. Inst. and Meth. in Phys Res. A 451 (2000) 265.

[8] O. Kofoed-Hansen, and K.O. Nielsen, K. Danske Vidensk. Selsk. Mat.-Fys. Medd. 26, No 7 (1951).

[9] J. R. J. Bennett et al., Nucl. Inst. and Meth. in Phys Res. B 126 (1997).

[10] J. Nolen et al., Liquid Lithium cooling for a 100kW ISOL and fragmentation targets To be published in Nuclear Phys. A.

[11] Proc. of the ICFA/ECFA Workshop “ Neutrino Factories based on Muon Storage Rings, Lyon, France, 5-9 July, 1999, Nucl. Inst. and Meth. in Phys Res. A451 1 (2000).

[12] Proc. of the International Workshop NuFACT'00 Muon Storage Ring for a Neutrino Factory, Montherey, CA, USA, 22-26 May, 2000 Nucl. Inst. and Meth. in Phys Res. A472 1 (2001).

[13] Proc. of the International Workshop NuFACT'01 Muon Storage Ring for a Neutrino Factory, Tsukuba, Japan 24-30 May 2001, To be published in, Nucl. Inst. and Meth. in Phys Res. A .

[14] M. Vretenar et al., Conceptual design of the SPL, a high-power super conducting $\mathrm{H}^{-}$linac at CERN. CERN 2000-012.

[15] H. L. Ravn, Phil. Trans. R. Soc. Lond. A 356 (1998) 1955.

[16] P. Drumm et al., Nucl. Inst. and Meth. in Phys Res. A472 627 (2001).

[17] R. Catherall, Radioactive Ion Beams Produced by Neutron-Induced Fission at ISOLDE, These proceedings. 
[18] P. Sievers, A stationary target for the CERN neutrino factory, To be published in Proc. of the International Workshop NuFACT'01 Muon Storage Ring for a Neutrino Factory, Tsukuba, Japan 24-30 May 2001, Nucl. Inst. and Meth. in Phys Res. A .

[19] J. Lettry et al., Nucl. Inst. and Meth. in Phys Res. B 126 (1997)170.

[20] H. Kirk et al., Target Studies with BNL E951 at the AGS" To be published in the proceedings of PAC 2001.

[21] A. Fabich, to be published in Proc. of the International Workshop NuFACT'02 Muon Storage Ring for a Neutrino Factory, Tsukuba, Japan 24-30 May 2001, To be published in, Nucl. Inst. and Meth. in Phys Res. A .

[22] H. L. Ravn, The CERN Target and Horn Concept For a Neutrino FactoryNucl. Inst. and Meth. in Phys Res. A.

[23] R.J. Weggel et al., Pion yield vs. geometry of target and $20 \mathrm{~T}$ pulse solenoid for a muon collider experiment. PAC 1999, p. 3047-3049.

[24] S. Van Der Meer, A directive device for charged particles and its use in an enhanced neutrino beam, CERN 61-7.

[25] A Ball, et. Al., Nucl. Inst. and Meth. in Phys Res. A451 359 (2000).

[26] B. Autin et al., Conducting Target, to be published in. Nucl. Inst. and Meth. in Phys Res. A.

[27] J. Lettry et al., Nucl. Instr. and Meth. in Phys Res B 126 (1997)130.

[28] H. L. Ravn et al., Nucl. Instr. and Meth. B 126 (1997) 176.

[29] U. Köster et al., Production and Use of Oxide Fibre Targets at ISOLDE, this conference. 


\begin{tabular}{|c|c|c|c|c|c|c|c|}
\hline \multirow[t]{2}{*}{$\begin{array}{l}\text { TARGET } \\
\text { TECHNIQUE }\end{array}$} & \multirow{2}{*}{$\begin{array}{c}\begin{array}{c}\text { Target } \\
\text { thickness } \\
\mathrm{g} / \mathrm{cm}^{2}\end{array}\end{array}$} & \multirow{2}{*}{$\begin{array}{l}\text { Cross } \\
\text { section } \\
\mathrm{cm}^{2}\end{array}$} & \multicolumn{2}{|c|}{$\begin{array}{l}\text { Proton driver } \\
\text { beam }\end{array}$} & \multirow{2}{*}{$\begin{array}{l}\text { Production } \\
\text { rate in target } \\
\text { atoms } / \mathrm{s}\end{array}$} & \multirow[t]{2}{*}{$\begin{array}{l}\text { Transfer } \\
\text { efficiency }\end{array}$} & \multirow{2}{*}{$\begin{array}{c}{ }^{6} \mathrm{He} \\
\text { Beam } \\
\text { ions/s }\end{array}$} \\
\hline & & & $(\mathrm{GeV})$ & $(\mathrm{mA})$ & & & \\
\hline $\mathrm{MgO}$ target technology presently operating & 3 & $1.0 \mathrm{E}-27$ & 1 & 0.004 & $1.8 \mathrm{E}+09$ & 0.025 & $4.6 \mathrm{E}+07$ \\
\hline $\mathrm{BeO}$ target improved with known technique & 30 & $1.0 \mathrm{E}-26$ & 1 & 0.1 & $4.6 \mathrm{E}+12$ & 0.25 & $1.1 \mathrm{E}+12$ \\
\hline New BeO target technology to be developed & 30 & $1.0 \mathrm{E}-26$ & 2.2 & 2.5 & $3.1 \mathrm{E}+14$ & 0.25 & $7.8 \mathrm{E}+13$ \\
\hline \multirow[t]{2}{*}{ Mercury-jet target to be developed } & 800 & $2.6 \mathrm{E}-26$ & 2.2 & 2.5 & $9.7 \mathrm{E}+14$ & 0.05 & $4.8 \mathrm{E}+13$ \\
\hline & \multicolumn{6}{|c|}{ Spallation neutron ( $n, \alpha$ p reaction } & \\
\hline $\begin{array}{l}\text { BeO with converter technology } \\
\text { development }\end{array}$ & 3 & $6.0 \mathrm{E}-25$ & 2.2 & 2.5 & $2.1 \mathrm{E}+15$ & 0.25 & $5.3 E+14$ \\
\hline
\end{tabular}




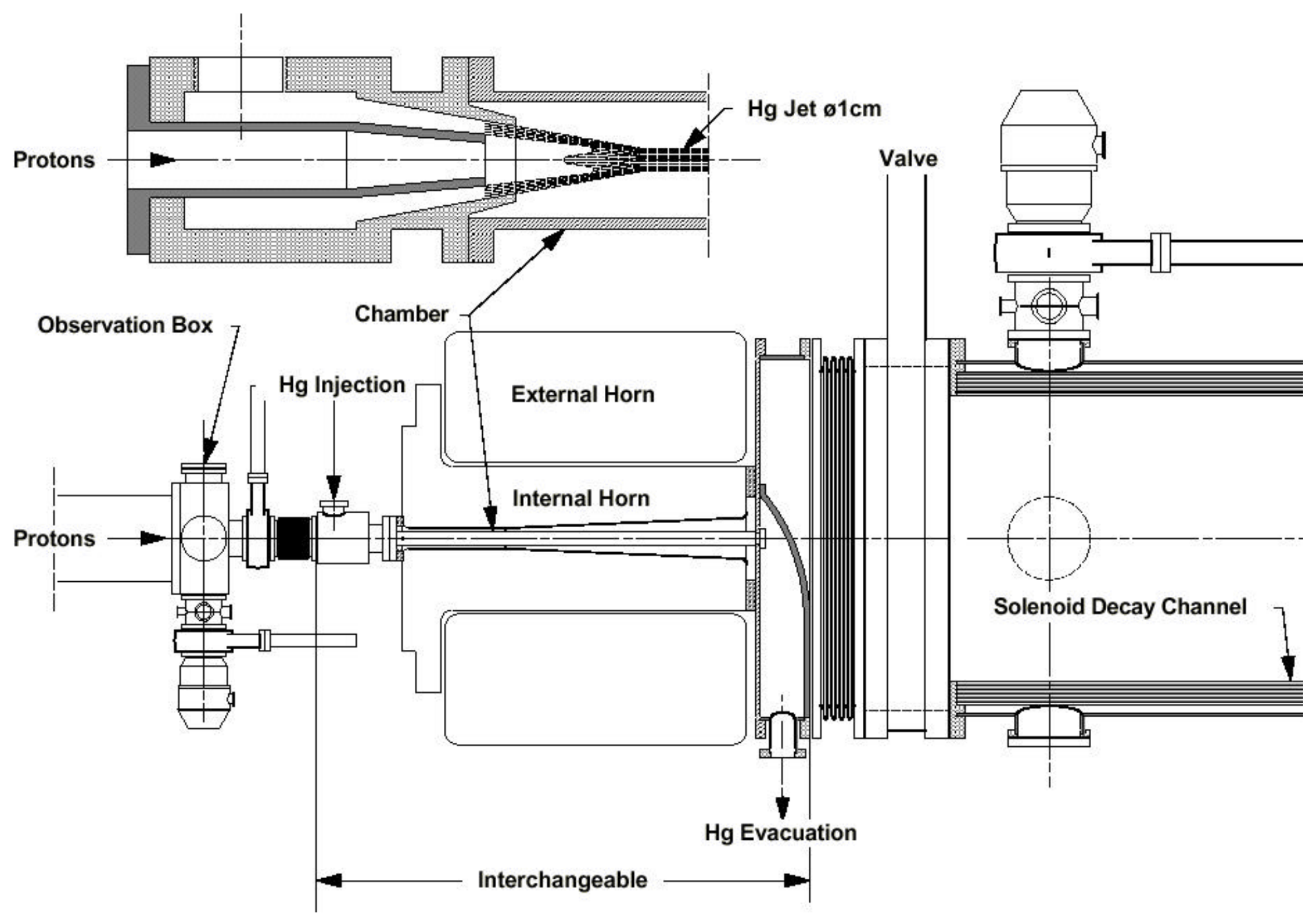

Figure caption

Figure 1.

Layout of a target and magnetic horn-module in which a spray of Mercury is directed into the neck region of the horn. The $\mathrm{Hg}$ is injected via an annular nozzle that allows coaxial injection of the proton beam. Also indicated is the double horn structure that serves to reduce the stress on the critical neck region. 